\title{
Reproducibility of a time-lapse embryo selection model based on morphokinetic data in a sequential culture media setting
}

\author{
Ender Yalçınkaya, Elif G. Ergin, Eray Çalışkan, Zeynep Öztel, Alev Özay, Hakan Özörnek \\ Eurofertil IVF Center, Istanbul, Turkey
}

Abstract

Objective: To compare the outcomes of embryos that were given a dynamic score based on a preconstructed embryo scoring model and to analyze whether this model complies with our data.

Material and Methods: A total of 910 transferred embryos with known implantation data were retrospectively analyzed in this study. All of the embryos were given a dynamic score based on the preconstructed hierarchical embryo scoring model.

Results: The highest pregnancy rate was seen in groups C+ and A- (48.2\% for each), and the lowest was observed in Group E (19.7\%). When implantation and clinical pregnancy rates were compared between groups, it was found that the highest and statistically significant implantation and clinical pregnancy rates were seen in group $\mathrm{C}+(32.7 \%$ for each, $\mathrm{p}=0.000)$. They were dropped down to $29.4 \%$ in Group A-

Conclusion: The outcomes of the embryos based on the dynamic score do not comply with the results of the preconstructed model. Each IVF laboratory is unique based on its practice. Therefore, we suggest that each IVF laboratory should determine its own embryo selection criteria based on its own data instead of using a preconstructed model. (J Turk Ger Gynecol Assoc 2014; 15: 156-60)

Key words: Preconstructed model, dynamic score, pregnancy rate

Received: 08 May, 2014

Accepted: 14 July, 2014

\section{Introduction}

The limitations in the number of embryos to be transferred by the new regulations have revealed the necessity of transferring the embryo with the highest implantation potential in order to increase pregnancy rate. There are many ongoing studies that aim to determine new noninvasive criteria to select the embryo with the highest implantation potential and thus to increase overall pregnancy rates (1-4).

The most important determinants of embryo quality are the morphological parameters of the developing embryos. During conventional assisted reproduction procedures, embryos are taken out of the incubators at limited time intervals in order to protect them against the negative effects of outside environmental conditions (temperature change, $\mathrm{pH}$ change, etc.) (5). Therefore, there are still some question marks in mind about embryo selection, since it is impossible to obtain detailed information about the developmental process of the embryos.

Time-lapse applications in assisted reproduction are based on the determination of embryo morphokinetics through monitoring by camera systems that are located in standard incubators for embryo culture. Improvements in these applications have led to the development of devices with time lapse monitoring as well as incubation properties. Recent studies related to time lapse incubation have reported interesting development pat- terns and promising results (6-8). Up to date, Meseguer et al. (7) have constructed a hierarchical model that helps choose the best embryo for transfer according to their experiences.

The purpose of this study is to compare the outcomes of the embryos with known implantation data based on their dynamic scores and to analyze whether a preconstructed embryo scoring model based on morphokinetic data can comply with our data.

\section{Material and Methods}

A total of 910 transferred embryos with known implantation data were retrospectively analyzed in this study. Ethics approval was obtained for the use of all relevant data. Characteristics of the embryos are given in Table 1. All patients underwent an antagonist protocol. In the fresh stimulation cycle of an antagonist regimen, gonadotropin administration was started on day 2-3 of the menstrual cycle. Follicular development was monitored by transvaginal ultrasound at least every 2 days after 5 days of gonadotropin administration. Gonadotropinreleasing hormone $(\mathrm{GnRH})$ antagonist (Cetrotide $0.25 \mathrm{mg}$; Merck Serono, Bari, Italy) was started on stimulation day 6, when the leading follicle reached a diameter of $13-14 \mathrm{~mm}$, and was used every day until human chorionic gonadotropin (hCG) administration. Finally, a single injection of 
Table 1. Demographic data of the embryos. The data are presented as numbers (percentages) or mean \pm standard deviation (SD) from the mean

\begin{tabular}{|c|c|c|c|c|c|c|c|c|c|c|}
\hline \multirow[b]{2}{*}{ Variable } & \multicolumn{9}{|c|}{ Dynamic score (based on Meseguer's model) } & \multirow{2}{*}{$\begin{array}{c}\mathbf{p} \\
\text { value }\end{array}$} \\
\hline & A+ & A- & B+ & B- & $\mathrm{C}+$ & C- & D+ & D- & $\mathbf{E}$ & \\
\hline Age (years) & $31 \pm 5.1$ & $30 \pm 4.7$ & $31.2 \pm 5.8$ & $31.8 \pm 5.3$ & $30.4 \pm 5.4$ & $31.4 \pm 5.9$ & $30.7 \pm 6.1$ & $32.2 \pm 5.6$ & $32.5 \pm 5.2$ & 0.08 \\
\hline BMI $\left(\mathrm{kg} / \mathrm{m}^{2}\right)$ & $25.3 \pm 4.0$ & $25 \pm 4.5$ & $24.8 \pm 3.3$ & $24.5 \pm 3.7$ & $25 \pm 4.3$ & $25.2 \pm 4.0$ & $24.8 \pm 3.3$ & $24.7 \pm 4.2$ & $25.4 \pm 4.9$ & 0.95 \\
\hline $\begin{array}{l}\text { Cause of infertility } \\
\text { anovulation }\end{array}$ & $14(6.6)$ & $6(7.1)$ & $5(6.1)$ & $2(5.9)$ & $24(9.6)$ & $4(6.5)$ & $11(11.8)$ & $1(3.2)$ & $6(9.8)$ & 0.09 \\
\hline Oligoasthenoteratospermia & $66(31.3)$ & 37 (43.5) & $31(37.8)$ & $14(41.2)$ & $75(29.9)$ & $21(33.9)$ & $37(39.8)$ & $14(45.2)$ & $26(42.6)$ & \\
\hline Azoospermia & $11(5.2)$ & $4(4.7)$ & $6(7.3)$ & $1(2.9)$ & $18(7.2)$ & $3(4.8)$ & $10(10.8)$ & $2(6.5)$ & $4(6.6)$ & \\
\hline Tubal factor & $33(15.6)$ & $3(3.5)$ & $10(12.2)$ & $1(2.9)$ & $24(9.6)$ & $10(16.1)$ & $14(15.1)$ & $1(3.2)$ & $8(13.1)$ & \\
\hline Unexplained infertility & $17(41.2)$ & 35 (41.2) & $30(36.6)$ & $16(47.1)$ & $110(43.8)$ & 24 (38.7) & $21(22.6)$ & $13(41.9)$ & 17 (27.9) & \\
\hline
\end{tabular}

hCG (Ovitrelle; Merck Serono, Bari, Italy) was administered to induce final follicular maturation as soon as three follicles of $\geq 17 \mathrm{~mm}$ were observed. The luteal phase was supported by micronized vaginal progesterone (Progynex ampule 200 mg; Farmako, İstanbul, Turkey), progesterone in oil (Progynex ampule $50 \mathrm{mg}$; Farmako, İstanbul, Turkey), or vaginal gel (Crinone $8 \%$ gel; Merck Serono, Bari, Italy). Progesterone supplementation was provided until the detection of clinical pregnancy or the 10th week of pregnancy at the discretion of the doctor in charge. In several cases, estrogen patch (Climara flaster 3.9; Schering, Germany) was started on the day of embryo transfer and continued until clinical pregnancy.

After controlled ovarian hyperstimulation, transvaginal ultrasound-guided oocyte retrieval was performed 36 hours after hCG injection. When follicle aspiration was finished, all oocytes were kept in culture for $2-4$ hours under the conditions of $37^{\circ} \mathrm{C}$, $6 \% \mathrm{CO}_{2}$, and $7 \% \mathrm{O}_{2}$ until denudation in the standard incubator. For all steps related to the embryo culture, two sequential culture media (Vitrolife; Göteborg, Sweden; and Medicult; Måløv, Denmark) were used. The oocytes were mechanically denuded 2-4 hours after oocyte pickup. Intracytoplasmic sperm injection (ICSI) procedure was performed for all metaphase II (MII) oocytes, and injected oocytes were placed into pre-equilibrated culture media. The fertilization check was performed at 16-18 hours after microinjection. Presence of two pronuclei was considered normal fertilization.

All fertilized oocytes were transferred into the wells of special dishes (EmbryoSlide; Unisense FertiliTech, Aarhus, Denmark) and placed into a time lapse incubator (EmbryoScope; Unisense FertiliTech, Aarhus, Denmark) until transfer under the conditions of $6.0 \% \mathrm{CO}_{2}, 7.0 \% \mathrm{O}_{2}$, and $37.0^{\circ} \mathrm{C}$. Day 2 transfers were excluded from the study. Only day 3 and day 5 transfers were included. All embryo transfers were performed between October 2012 and December 2013 at the Eurofertil IVF Center, Istanbul, Turkey. Dynamic scores of the embryos were determined based on a preconstructed hierarchical embryo scoring model by Meseguer et al. (7). The morphology of two day 3 transfer embryos of different dynamic scores is shown in Figure 1.
Table 2. Dynamic score classification of Meseguer et al. (7) based on cleavage timings

\begin{tabular}{|c|c|}
\hline Category & Criteria (timings) \\
\hline$A+(*)$ & t5 (within 48.8-56.6 h); s2 $\leq 0.76 \mathrm{~h} ; \mathrm{cc} 2 \leq 11.9 \mathrm{~h}$ \\
\hline A- & t5 (within 48.8-56.6 h); s2 $\leq 0.76 \mathrm{~h} ; \mathrm{cc} 2>11.9 \mathrm{~h}$ \\
\hline $\mathrm{B}+$ & t5 (within $48.8-56.6$ h); s2 >0.76 h; cc2 $\leq 11.9 \mathrm{~h}$ \\
\hline B- & t5 (within $48.8-56.6$ h); s2 >0.76 h; cc2>11.9 h \\
\hline $\mathrm{C}+$ & t5 (outside $48.8-56.6 \mathrm{~h}$ ); s2 $\leq 0.76 \mathrm{~h} ; \mathrm{cc} 2 \leq 11.9 \mathrm{~h}$ \\
\hline C- & t5 (outside $48.8-56.6 \mathrm{~h}$ ); s2 $\leq 0.76 \mathrm{~h} ; \mathrm{cc} 2>11.9 \mathrm{~h}$ \\
\hline $\mathrm{D}+$ & t5 (outside $48.8-56.6 \mathrm{~h}$ ); $\mathrm{s} 2>0.76 \mathrm{~h} ; \mathrm{cc} 2 \leq 11.9 \mathrm{~h}$ \\
\hline D- & t5 (outside $48.8-56.6 \mathrm{~h}$ ); s2>0.76 h; cc2>11.9 h \\
\hline $\mathrm{E}$ & $\begin{array}{l}\text { Uneven blastomere size at } 2 \text {-cell stage } \\
\text { Abrupt division from } 1 \text { to } 3 \text { or more cells } \\
\text { Multinucleation at } 4 \text {-cell stage }\end{array}$ \\
\hline \multicolumn{2}{|c|}{$\begin{array}{l}\text { *Meseguer et al. (7) selected transfer embryos based on their mor- } \\
\text { phological grading, and they found that Group A+ had the highest } \\
\text { implantation potential }\end{array}$} \\
\hline
\end{tabular}

\section{Statistical Analysis}

The statistical analysis of the data was performed by using Statistical Package for Social Sciences 16.0 (SPSS Inc, Chicago, IL, USA). The continuous variables were analyzed for normality distribution with Kolmogorov-Smirnoff test. All continuous variables analyzed were normally distributed and were compared between the groups by using the analysis of variance test (ANOVA). The categorical data were compared between the two groups by using chi-square test. For all comparisons, probability $\mathrm{p}<0.05$ was considered to be statistically significant.

\section{Results}

The dynamic score classification by Meseguer et al. (7) is given in Table 1. Demographic data regarding the embryos are presented in Table 2. While mean age and body mass index (BMI) were similar between groups, there was a statistically significant difference in terms of total follicle-stimulating hormone (FSH) 

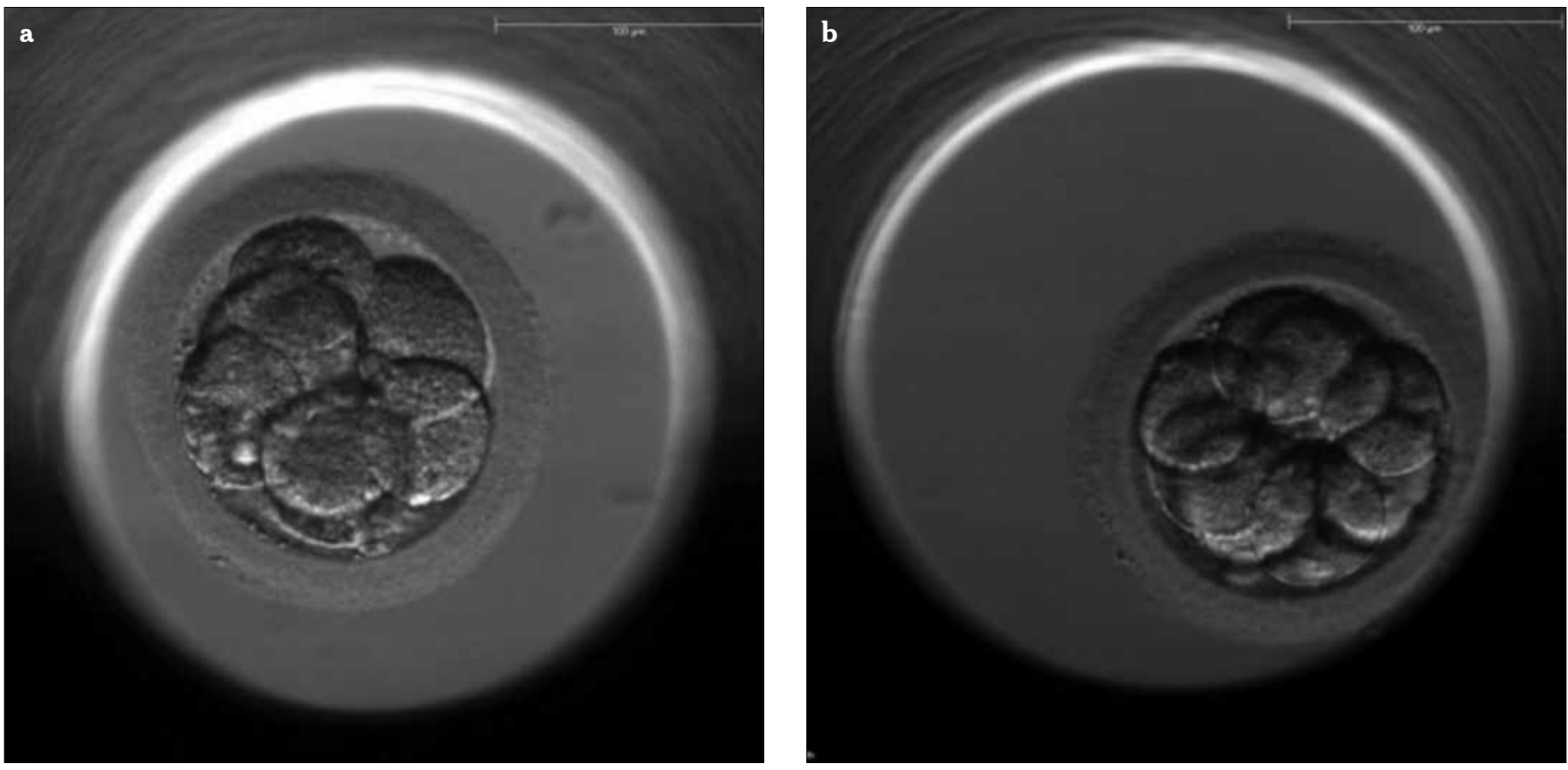

Figure 1. a, b. Figures above are two examples of transfer embryos at day 3 . The photographs were captured by the camera system in timelapse system. Embryo morphology looks similar; but dynamic scores based on embryo morphokinetics are different. Embryo with a dynamic score of A+ (a), Embryo with a dynamic score of C+ based on Meseguer's scoring model (b)

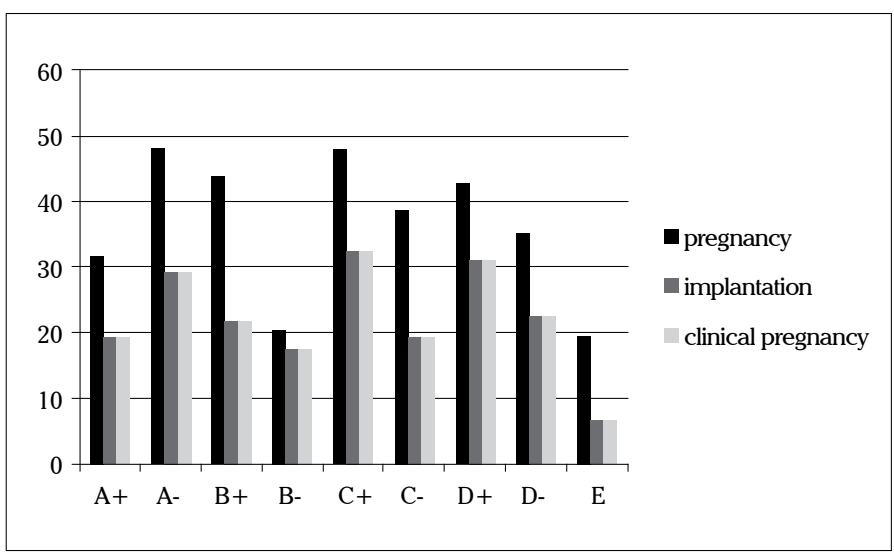

Figure 2. Comparison of the clinical outcomes of the embryos with different dynamic scores $(\mathbf{p}<0.05)$

dose and number of oocytes between all groups $(\mathrm{p}=0.002$ and $\mathrm{p}=0.003$, respectively). When group $\mathrm{E}$ was excluded from the study, it was observed that there was no statistically significant difference between groups in terms of mean age, BMI, total FSH dose, and number of oocytes collected ( $\mathrm{p}>0.05)$.

Cycle characteristics and clinical outcomes are given in Table 3, and clinical outcomes are also shown in Figure 2. The highest pregnancy rates were seen in groups $\mathrm{C}+$ and A- $(48.2 \%$ for each), and the lowest was observed in Group E (19.7\%). When implantation and clinical pregnancy rates were compared, it was found that the highest and statistically significant implantation and clinical pregnancy rates were seen in group $\mathrm{C}+(32.7 \%$ for each, $p=0.000$ ). They were dropped down to $29.4 \%$ in Group A-. The lowest rates were again seen in Group E (6.6\% per each).
When parameters, such as the type of gonadotropin used and cause of infertility, were compared based on clinical pregnancy within each group, it was observed that there were no statistically significant differences between different types of gonadotropins and causes of infertility in terms of clinical pregnancy ( $p>0.05$ for each). In addition, no statistically significant difference was found between groups in terms of embryo culture media and luteal support used ( $p>0.05$ ).

When Group E was excluded from the analysis, it was again found that $\mathrm{C}+$ had significantly higher pregnancy and implantation rates compared to other groups $(p=0.003$ and $p=0.026$, respectively). When BMI, total FSH dose, number of oocytes aspirated, and age were compared between groups except group E, no significant differences were observed $(p=0.946$, $\mathrm{p}=0.140, \mathrm{p}=0.270$, and $\mathrm{p}=0.314$, respectively).

\section{Discussion}

Time-lapse microscopy has become a powerful technology for the study of early embryonic development in recent years. This is apparent by the publication of an increasing number of studies on this subject. Using this technique, a variety of morphological and dynamic parameters can be extracted from individual embryos and potentially used as predictive markers for healthy embryo development and used to investigate many unknown developmental questions (9). These predictive markers have gained more importance, especially in countries where singleembryo transfer is implemented, and thus, it is crucial to select the best embryo for transfer. These systems have revealed that even embryos with the same morphology on day 3 might have shown different cleavage patterns during development. 
Table 3. Cycle characteristics and clinical outcomes

\begin{tabular}{|c|c|c|c|c|c|c|c|c|c|c|}
\hline \multirow[b]{2}{*}{ Variable } & \multicolumn{9}{|c|}{ Dynamic score (based on Meseguer's model) } & \multirow{2}{*}{$\begin{array}{c}p \\
\text { value }\end{array}$} \\
\hline & A+ & A- & B+ & B- & $\mathrm{C}+$ & C- & $\mathrm{D}+$ & D- & $\mathbf{E}$ & \\
\hline Total FSH dose (IU) & $2598 \pm 1009$ & $2394 \pm 1009$ & $2557 \pm 1639$ & $2515 \pm 1041$ & $2416 \pm 966$ & $2779 \pm 1124$ & $2507 \pm 919$ & $2853 \pm 1224$ & $3105 \pm 1626$ & $0.002^{*}$ \\
\hline $\begin{array}{l}\text { Number of oocytes } \\
\text { aspirated }\end{array}$ & $12.7 \pm 6.5$ & $12.5 \pm 7.0$ & $12.2 \pm 6.6$ & $10.3 \pm 5.4$ & $12.4 \pm 6.4$ & $12.3 \pm 7.0$ & $11.6 \pm 6.5$ & $10 \pm 5.8$ & $9 \pm 5.8$ & $0.003^{*}$ \\
\hline \multicolumn{11}{|l|}{ Culture media used } \\
\hline Vitrolife & $109(51.7)$ & $51(60)$ & $46(56.1)$ & $21(61.8)$ & $117(46.6)$ & $33(53.2)$ & $44(47.3)$ & $20(64.5)$ & $37(60.6)$ & 0.12 \\
\hline Medicult & $102(48.3)$ & $34(40)$ & $36(43.9)$ & $13(38.2)$ & $134(53.4)$ & $29(46.8)$ & $49(52.7)$ & $11(35.5)$ & $24(39.4)$ & \\
\hline \multicolumn{11}{|l|}{ Luteal support } \\
\hline Prog.* & $97(46)$ & $48(56.5)$ & $36(43.9)$ & $10(29.4)$ & $138(55)$ & $41(66.1)$ & $52(62.7)$ & $17(54.8)$ & $24(39.3)$ & 0.34 \\
\hline Prog+E2** & $114(54)$ & 37 (43.5) & $46(56.1)$ & $24(70.6)$ & $113(45)$ & $21(33.9)$ & $31(37.3)$ & $14(45.2)$ & $37(60.7)$ & \\
\hline \multicolumn{11}{|l|}{ Clinical outcomes } \\
\hline Positive bhcg (\%) & 31.8 & 48.2 & 43.9 & 20.6 & 48.2 & 38.7 & 43 & 35.5 & 19.7 & $0.00 *$ \\
\hline $\begin{array}{l}\text { Implantation rate } \\
(\%)\end{array}$ & 19.4 & 29.4 & 22 & 17.6 & 32.7 & 19.4 & 31.2 & 22.6 & 6.6 & $0.00 *$ \\
\hline $\begin{array}{l}\text { Clinical pregnancy } \\
\text { rate (\%) }\end{array}$ & 19.4 & 29.4 & 22 & 17.6 & 32.7 & 19.4 & 31.2 & 22.6 & 6.6 & $0.00 *$ \\
\hline
\end{tabular}

An incubation system with a time-lapse microscopy setting has two parts that may offer different advantages. The first part is a microscopy system that acquires continuous monitoring of the embryos, and the other is an incubation system that remains stable during data acquisition.

Time-lapse microscopy seems to offer many advantages over traditional time-point microscopy. While traditional time-point microscopy provides images at distinct time points, a time-lapse system generates continuous imaging until embryo transfer. Besides, it is connected to imaging software, and it analyzes the images by different observers, therefore decreasing inter- and intraobserver variability, since it offers repeatability of the saved images $(4,7)$. But, again, some variations were also seen in some time-lapse annotations in a study by Sundvall et al. (10).

The second part is the incubation stability. In a large-scale retrospective analysis by Meseguer et al. (7) comparing clinical pregnancy rates with time-lapse and standard incubation (SI), it was found that there was a significantly increased clinical pregnancy rate over those using SI. In this study, they declared the advantages of time-lapse incubation as less handling of embryos and no need for intermediate observations, which would reduce the risk of loss or contamination within the laboratory (11).

The main goal of using a time-lapse system is to be able to create a mathematical model of a scoring system from all data obtained from the embryos. For this reason, it is crucial to analyze all embryos in detail and keep the records of the clinical outcomes of each embryo. The purpose of this model is to be able to select the embryo with the highest implantation potential as early as possible. It was shown by many authors that blastocyst-stage embryo transfer was shown to increase the chance of pregnancy compared to day 3 embryo transfers
$(12,13)$. Additional data suggest that prolonged in vitro culture may lead to imprinting errors and subsequent epigenetic disorders $(14,15)$. Because of these possible risks of extended embryo culture, a time-lapse monitoring system seems to offer the construction of an embryo scoring system that may lead the laboratory to select the embryo for transfer at an early developmental stage.

Many authors have reported different prognostic factors for better embryonic growth, and all of these parameters have focused mostly on developmental processes before day 3 , and they suggested that the quality of the embryo was predetermined before embryonic gene activation $(4,7,8)$. Meseguer et al. (7) constructed the first embryo scoring model that gives a dynamic score, varying between $\mathrm{A}+$ to $\mathrm{F}$, to each embryo based on the correlation between several developmental timings and the clinical pregnancy outcomes they present. According to their model, embryos with a dynamic score of $\mathrm{A}+$ had the highest implantation potential compared to the others. In our study, we used Meseguer's model to classify our embryos that were transferred, and our results showed that $\mathrm{C}+$ plus embryos had the highest implantation potential compared to the others, showing that the model constructed by Meseguer et al. (7) does not comply with our data. There may be some possible reasons for that difference, depending on the laboratory conditions and techniques used.

First of all, Meseguer et al. (7) used single-step culture media, and we used sequential culture media for embryo culture. In the study by Basile et al. (16) comparing two types of culture media, they did not find any significant differences between them. Ciray et al. (17) also compared two different types of culture media in their study and found that the embryos that were 
cultured in single-step media reached the 2- to 5-cell stages earlier compared to the ones in sequential media. However, clinical pregnancy rates were similar between groups. Although it does not change overall outcome, it may cause a change in the scoring model, since it has the possibility of changing embryo kinetics. This may be a possible reason why our results based on the dynamic scores of Meseguer's model did not give the same morphokinetic outcomes.

Secondly, they were not using an $\mathrm{O}_{2}$-controlled time-lapse incubation system. There are several studies evaluating the effect of oxygen on embryonic development and kinetics in the literature. Based on the results of these studies, it is now known that high oxygen levels during culture have a negative effect on embryonic development. Kirkeegaard et al. (18) evaluated the effect of oxygen concentration on embryos and evaluated their development by time-lapse monitoring. According to the results of their study, it was found that the timing of the third cleavage cycle (division to 8 cells) for embryos that were cultured under high oxygen concentrations was delayed. Wale and Gardner performed a similar study in mouse embryos and observed that oxygen can influence mouse embryo development at both the cleavage stage and post-compaction stages (19). In our study, we used 7\% $\mathrm{O} 2$ for the embryo culture, and it is possible that this level could have affected the kinetics of our embryos in a different pattern compared to Meseguer's model.

In our study, the lowest clinical pregnancy rate was observed in group E, complying with Meseguer's model. This is an expected outcome, since group E was composed of embryos that had not shown a proper cleavage pattern. There were significant differences between all groups in terms of total FSH dose and number of oocytes aspirated. In order to see if group $\mathrm{E}$ created this difference, we made another analysis by excluding group E. Based on this analysis, it was found that both parameters did not show any statistical difference between other groups, suggesting that group E creates this confounding effect and includes the embryos of patients who were poor responders or advanced in age who needed more FSH doses.

Our results showed that variations in different laboratories may result in different embryo developmental criteria. Thus, we suggest that each IVF laboratory should determine its own embryo selection criteria based on its own data instead of using a preconstructed model.

Ethics Committee Approval: Ethics committee approval was received for this study.

Informed Consent: Written informed consent was obtained from patients who participated in this study.

Peer-review: Externally peer-reviewed.

Author contributions: Concept - E.Ç., E.G.E., E.Y.; Design - E.Ç., E.G.E, E.Y.; Supervision - H.Ö., A.Ö.; Resource - Z.Ö., E.Y., E.G.E. ; Materials - E.Y., Z.Ö.; Data Collection\&/or Processing - Z.Ö., E.Y.; Analysis\&/or Interpretation - E.Ç., E.Y.; Literature Search - E.G.E., E.Y.; Writing - E.Y.; Critical Reviews - A.Ö., H.Ö.

Conflict of Interest: No conflict of interest was declared by the authors.

Financial Disclosure: The authors declared that this study has received no financial support.

\section{References}

1. Seli E, Sakkas D, Scott R, Kwok SC, Rosendahl SM, Burns DH. Noninvasive metabolomic profiling of embryo culture media using Raman and near-infrared spectroscopy correlates with reproductive potential of embryos in women undergoing in vitro fertilization. Fertil Steril 2007; 88: 1350-7. [CrossRef]

2. Sakkas D, Gardner DK. Noninvasive methods to assess embryo quality. Curr Opin Obstet Gynecol 2005; 17: 283-8. [CrossRef]

3. Katz-Jaffe MG, Gardner DK. Symposium: Innovative techniques in human embryo viability assessment. Can proteomics help to change the future of human assisted conception? Reprod Biomed Online 2008; 17: 497-501. [CrossRef]

4. Wong CC, Loewke KE, Bossert NL, Behr B, De Jonge CJ, Baer TM, Reijo Pera RA. Non-invasive imaging of human embryos before embryonic genome activation predicts development to the blastocyst stage. Nat Biotechnol 2010; 28: 1115-21. [CrossRef]

5. Wong C, Chen AA, Behr B, Shen S. Time-lapse microscopy and image analysis in basic and clinical embryo development research. Reprod Biomed Online 2013; 26: 120-9. [CrossRef]

6. Hashimoto S, Kato N, Saeki K, Morimoto Y. Selection of highpotential embryos by culture in poly(dimethylsiloxane) microwells and time-lapse imaging. Fertil Steril 2012; 97: 332-7. [CrossRef]

7. Meseguer M, Herrero J, Tejera A, Hiligsoe KM, Ramsing NB, Remohi J. The use of morphokinetics as a predictor of embryo implantation. Hum Reprod 2011; 26: 2658-71. [CrossRef]

8. Lemmen JG, Agerholm I, Ziebe S. Kinetic markers of human embryo quality using time-lapse recordings of IVF/ICSI-fertilized oocytes. Reprod Biomed Online 2008; 17: 385-91. [CrossRef]

9. Dal Canto M, Coticchio G, Mignini Renzini M, De Ponti E, Novara PV, Brambillasca F, et al. Cleavage kinetics analysis of human embryos predicts development to blastocyst and implantation. Reprod Biomed Online 2012; 25: 474-80. [CrossRef]

10. Sundvall L, Ingerslev HJ, Knudsen UB, Kirkegaard K. Inter- and intra-observer variability of time-lapse annotations. Hum Reprod 2013; 28: 3215-21. [CrossRef]

11. Meseguer M, Rubio I, Cruz M, Basile N, Marcos J, Requena A. Embryo incubation and selection in a time-lapse monitoring system improves pregnancy outcome compared with a standard incubator: A retrospective cohort study. Fertil Steril 2012; 981 481-9.

12. Papanikolaou EG, Camus M, Kolibianakis EM, Van Landuyt L, Van Steirteghem A, Devroey P. In vitro fertilization with single blastocyst-stage versus single cleavage-stage embryos. N Engl J Med 2006; 354: 1139-46. [CrossRef]

13. Blake D, Farquhar C, Johnson N, Proctor M. Cleavage stage versus blastocyst stage embryo transfer in assisted reproductive technology. Cochrane Database Syst Rev 2007; CD002118.

14. Niemitz EL, Feinberg AP. Epigenetics and assisted reproductive technology: A call for investigation. Am J Hum Genet 2004; 74: 599-609. [CrossRef]

15. Manipalviratn S, DeCherney A, Segars J. Imprinting disorders and assisted reproductive technology. Fertil Steril 2009; 91: 305-15. [CrossRef]

16. Basile N, Morbeck D, Garcia-Velasco J, Bronet F, Meseguer M. Type of culture media does not affect embryo kinetics: a time lapse analysis of sibling oocytes. Hum Reprod 2013; 28: 634-41. [CrossRef]

17. Ciray HN, Aksoy t, Goktas C, Ozturk B, Bahceci M. Time-lapse evaluation of human embryo development in single versus sequential culture media--a sibling oocyte study. J Assist Reprod Genet 2012; 29: 891-900. [CrossRef]

18. Kirkegaard K, Hindkjaer JJ, Ingerslev HJ. Effect of oxygen concentration on human embryo development evaluated by time-lapse monitoring. Fertil Steril 2013; 99: 738-44. [CrossRef]

19. Wale PL, Gardner DK. Time-lapse analysis of Mouse embryo development in oxygen gradients. Reprod Biomed Online 2010; 21: 402-10. [CrossRef] 